\title{
Urothelial carcinoma: the evolving landscape of immunotherapy for patients with advanced disease
}

This article was published in the following Dove Press journal: Research and Reports in Urology

\author{
Brian Dietrich \\ Sandy Srinivas ${ }^{2}$ \\ 'Department of Hematology/ \\ Oncology, Stanford University School \\ of Medicine/Stanford Cancer Center, \\ Stanford, CA, USA; ${ }^{2}$ Department of \\ Oncology, Stanford University School \\ of Medicine/Stanford Cancer Center, \\ Stanford, CA, USA
}

\begin{abstract}
Urothelial carcinoma is the sixth most common malignancy in the US. While most patients present with non-muscle-invasive disease, many will develop recurrent disease including some progressing to muscle invasive metastatic cancer. Treatment outcomes have remained poor and stagnant for those with more advanced illness, with typical 5-year survival rates in the range of $\leq 15 \%$. While first-line, platinum-based chemotherapy remains the current standard for those eligible, the recent incorporation of checkpoint inhibitors into the management of advanced bladder cancer has resulted in an expansion of treatment options for a difficult-to-treat disease. This review will discuss the historic standard treatment options, followed by the more recent evolving role immune therapy has in the management of bladder cancer.
\end{abstract}

Keywords: urothelial, immunotherapy, checkpoint inhibitor, bladder cancer

\section{Introduction}

Bladder cancer (with urothelial carcinoma accounting for $90 \%$ of bladder tumors in the USA) is the most common malignancy of the urinary tract system and the sixth most common cancer in the USA, with an estimated 79,030 cases to be diagnosed in 2017. ${ }^{1,2}$ The incidence of disease is four times greater in men than in women, with 60,490 men and 18,540 women diagnosed annually. ${ }^{1,2}$ Although most cases are non-muscle invasive at diagnosis, nearly $70 \%$ will have a recurrence or new occurrence within 5 years, with some patients developing more advanced muscle-invasive or metastatic disease, in the range of $10 \%-20 \% .{ }^{3}$ For patients diagnosed with the disease, 5 -year survival rates are around $77 \%$ for all stages combined, and $<15 \%$ for those in the metastatic setting, despite multidisciplinary therapeutic advances. ${ }^{4}$ Further, given that most bladder cancers are diagnosed in an elderly population (median age : 73.2 years) with possible comorbidities, standard systemic options with cisplatin may not be safe and may be absolutely contraindicated for a significant number of newly diagnosed patients. Given the continued stagnant outcomes for patients with locally advanced and metastatic disease, there remains an unmet need for effective interventions to improve upon these numbers.

The hallmark of cancer as a biologic entity stems from its ability to acquire a multitude of survival mechanisms during its development, including the potential to evade immune recognition. The concept of immunotherapy in bladder cancer dates back $>40$ years to the 1960s, when use of intravesical bacillus Calmette-Guerin (BCG) in nonmuscle-invasive bladder cancer first demonstrated immune-mediated therapeutic effects
Correspondence: Sandy Srinivas Department of Oncology, Stanford University School of Medicine/Stanford Cancer Center, 875 Blake Wilbur Drive, Stanford, CA 94305, USA

Email sandysri@stanford.edu 
via the triggering of a variety of localized immune responses which may persist for extended periods of time. ${ }^{5}$ In 1990, the US Federal Drug Administration (FDA) approved the use of BCG for the treatment of carcinoma in situ of the bladder and for the prophylaxis of recurrent tumors following transurethral resection. Since that time, there has been an explosion of interest in the field of immunotherapy, which continues to evolve. In recent years, the greatest focus of research has been conducted on part of a family of checkpoint inhibitors, programmed death 1 (PD-1) and its ligand counterpart programmed death-ligand 1 (PD-L1). PD-L1 is variably expressed in a variety of tumor cells and binds with PD-1 on T cells and other immune cells when they are activated. ${ }^{6}$ This interaction results in dampening and suppression of the immune response, allowing tumors to grow unchecked. By blocking this inhibitory effect through the use of directed antibodies, exploitation of this pathway can eradicate some of the evasive measures employed by cancer and allow for increased immune surveillance and immune destruction of tumors. Use of this strategy has been demonstrated to be an effective treatment option in progressive, advanced urothelial carcinoma, with FDA approval of five different immunotherapy agents for use since May 2016, revolutionizing the treatment of patients with advanced disease. Here, we will first review historic standard treatment options, followed by the more recent evolving role immune therapy has in the management of bladder cancer. The scope of this review will be limited to patients with locally advanced or metastatic disease.

\section{Systemic therapy for advanced disease}

Several chemotherapy agents have demonstrated activity in urothelial cancers, with a number of various drug combinations used acting through different mechanisms. In the 1990s, clinical trials demonstrated the superiority of the four-drug MVAC (methotrexate, vinblastine, doxorubicin, cisplatin) regimen when compared with CISCA (cyclophosphamide, doxorubicin, cisplatin), showing a 12.5 months median overall survival (OS) compared with 8.2 months in patients with metastatic disease and a greater overall response rate (ORR). ${ }^{7}$ Toxicity is a major concern with MVAC therapy, especially given that many of the patients are older adults with medical comorbidities. Some early series had up to $54 \%$ of patients requiring hospitalization for neutropenic sepsis while on therapy. ${ }^{8}$ The implementation of growth factor support with growth-colony stimulating factor, however, has significantly reduced the rates of highgrade toxicity, including neutropenia, sepsis, and mucositis. ${ }^{9}$ In comparison to standard MVAC, dose-dense administration with growth factor support results in better outcomes and tolerability and is considered one standard of care for patients with adequate renal function and good performance status. ${ }^{10}$ The combination of gemcitabine and cisplatin (GC) has been shown to have similar efficacy (ORR 49\% GC vs 46\% MVAC; median OS 14 months GC vs 15 months MVAC) and less toxicity in comparison to MVAC in a Phase III trial, though this study was not designed as a non-inferiority trial. ${ }^{11}$ Given their proven efficacy, both dose-dense MVAC and GC are considered standard regimens for metastatic disease for patients who are cisplatin eligible and are recommended by the National Comprehensive Cancer Network. ${ }^{2}$

Due to comorbidities, a substantial number of patients may not be eligible for cisplatin-based treatment. The superiority of cisplatin to carboplatin-based chemotherapy has been demonstrated in multiple Phase II clinical studies. ${ }^{12}$ Proposed criteria to determine ineligibility of a cisplatin-based regimen include at least one of the following: poor performance status (ECOG 2 or higher), $\mathrm{CrCl}<60 \mathrm{~mL} / \mathrm{min}$, hearing loss or neuropathy that are grade 2 or worse, and/or New York Heart Association class III heart failure. ${ }^{13}$ If the basis of ineligibility is renal impairment, it is critical to ascertain the cause of this dysfunction prior to starting treatment, as some patients may have an obstructive etiology that may be effectively addressed with procedural interventions such as nephrostomy tubes. For patients who are ineligible for cisplatin-based treatment but are otherwise fit for chemotherapy, carboplatin combination regimens may be considered. The Phase II/III European Organization for the Research and Treatment of Cancer (EORTC) study evaluated 238 chemotherapy naïve patients with baseline poor performance status or inadequate renal function, randomly assigning them to receive either carboplatin and gemcitabine versus methotrexate, vinblastine, and carboplatin (M-CAVI). ${ }^{14}$ The overall results of the study suggest similar efficacy with carboplatin/gemcitabine compared to M-CAVI, with a higher ORR (41\% for Carbo/Gem vs $30 \%$ for M-CAVI) that did not reach statistical significance and no difference in median OS (9 months with carbo/gem vs 8 months with M-CAVI) or median progression-free survival (PFS; 6 vs 4 months). Neutropenia occurred less frequently in the carbo/gem arm, though a higher incidence of serious thrombocytopenia was seen. ${ }^{14}$ Although response rates are less than with cisplatin-containing regimens, the combination of gemcitabine and carboplatin remains a treatment consideration for patients with comorbidities that preclude other therapy options.

Several non-platinum regimens have been studied, both single agent and combination studies with some encouraging results. The combination of paclitaxel plus gemcitabine 
given at various dosing schedules has demonstrated overall response ranging from $54 \%$ to $70 \%{ }^{15-18}$ and median survival rates up to 16 months. ${ }^{15-18}$ Paclitaxel seems to have slightly greater activity than docetaxel. Two Phase II trials to date have evaluated the combination of docetaxel with gemcitabine in the up-front setting, showing overall responses of 33\% and $52 \%$, with median survival up to 15 months with the combination. ${ }^{19,20}$ There have been evaluations of several single-agent chemotherapy drugs in the metastatic setting, including ifosfamide, gemcitabine, paclitaxel, docetaxel, and doxorubicin. ${ }^{21-27}$ Use of single agents has been evaluated in both the first-line setting and in previously treated patients. Responses, however, are generally short lived without consistent improvement in OS rates. Given the lower response rates, use of single agents is typically not preferred if patients can tolerate combination regimens. Triplet regimens have also been used, though they have not been shown to be convincingly better than most two-drug combinations. In the International EORTC 30987 study, patients with advanced disease who had not received prior systemic therapy were randomized to receive either GC or the triplet combination of paclitaxel, cisplatin, and gemcitabine (PCG). Though the triplet regimen yielded a higher overall response rate $(56 \%$ vs $44 \%$ for GC), there was trend toward improvement in PFS and OS that did not reach statistical significance. ${ }^{28}$

Despite decent initial response rates, patients with advanced disease invariably progress and may be eligible for further therapy with alternate regimens. For patients who are not candidates for immune-based therapies or have progress on prior immunotherapy treatments, second-line chemotherapies have been studied, though there is no standard second-line chemotherapy option. Several single-agent considerations are available based on data from Phase II studies. ${ }^{29}$ Pemetrexed is an antifolate chemotherapy drug with some efficacy in Phase II studies enrolling previously treated patients. In the Hoosier Oncology Group study, single-agent overall response rates (ORRs) as a second-line therapy in 47 patients were $28 \%$ including three complete responses (CRs), with a median OS of 10 months. ${ }^{30}$ In another Phase II study of 48 patients treated with nab-paclitaxel, overall response rates were $27 \%$, including one CR $(2 \%)$ and 12 partial responses (PRs; $25.5 \%$ ) after a median of six cycles. ${ }^{31}$ In a randomized Phase III study enrolling 370 patients with progressive disease after a platinum-containing regimen, treatment with vinflunine, a microtubule inhibitor, versus best supportive care (BSC) alone demonstrated a low ORR of $9 \%$ with a small OS benefit of 2.3 months ( 6.9 months for vinflunine and $\mathrm{BSC}$ vs 4.6 months for BSC alone). ${ }^{32}$ Based on these data, vinflunine was approved for use in Europe, though not in the USA likely due to associated toxicities with the therapy (neutropenia in 50\%, febrile neutropenia rates of $6 \%$ ) and the low response rates observed. In the Phase III RANGE trial, patients with platinum refractory disease were randomized to receive the vascular endothelial growth factor receptor-2 antibody ramucirumab in combination with docetaxel or docetaxel plus placebo. ${ }^{33}$ PFS was double in the combination arm to 4.1 months compared with 2.8 months with single-agent docetaxel. ORRs were also higher in the combination $(24.5 \%$ vs $14 \%)$. The most common side effects included fatigue, alopecia, diarrhea, and nausea, with rare fatal events of neutropenic sepsis reported in patients allocated to ramucirumab. ${ }^{33}$ Longer follow-up analysis is needed to determine whether a survival benefit is seen, as well as information on activity following treatment with immunotherapy drugs, which was allowed in the study. As of the writing of this article, ramucirumab is currently not approved for the treatment of patients with advanced disease.

\section{Checkpoint inhibitors for advanced disease}

In recent years, there have been a multitude of applications for novel immune therapies in treating a variety of malignancies, including bladder cancer. The advent of T-cell checkpoint inhibition has changed the treatment landscape in this disease, given the significant percentage of patients who are cisplatin ineligible or have progressive disease following platinumbased therapy. Bladder cancer is a very heterogenous disease, with a rather high frequency of somatic mutations,${ }^{34}$ on par with other disease processes such as melanoma and non-small cell lung cancer. In fact, urothelial carcinoma has the third highest mutation burden across different tumor types. ${ }^{35}$ The hypermutational phenotype that develops in malignancies such as urothelial carcinoma leads to an increase in neoantigen burden, which is hypothesized to correspond to a tumor's sensitivity to immune checkpoint blockade that can be exploited by immune therapy drugs. ${ }^{36,37} \mathrm{PD}-1$ and its ligand counterpart PD-L1 are the most common targets of currently approved therapeutic interventions. Activation of these receptors impedes T-cell activation through signaling mechanisms, which ultimately leads to downregulation of immune recognition. Although chemotherapy regimens are still considered to be the best first-line approach for most eligible patients, PD-L1 and PD-1 inhibitors may yield more durable responses than conventional chemotherapy and continue to show promising results as new treatment options. Since May 2016, the FDA has approved five different immunotherapy agents for 
use in urothelial carcinoma, all of which are inhibitors of PD-1 or PD-L1: atezolizumab (Tecentriq), pembrolizumab (Keytruda), nivolumab (Opdivo), durvalumab (Imfinzi), and avelumab (Bavencio). Of these, only atezolizumab and pembrolizumab are approved as first-line therapy for patients ineligible to receive cisplatin chemotherapy, while all the agents are approved in previously treated patients (Table 1).

\section{Atezolizumab (Tecentriq, Genentech, Inc)}

Atezolizumab is an Fc-engineered, humanized, monoclonal antibody that targets PD-L1, inhibiting the interaction with PD-1 and B7.1 receptors. ${ }^{38}$ Approval of the drug for use in the metastatic setting after prior therapy with a platinumbased regimen occurred in May 2016 based on the results of cohort 2 of the single-arm, open-label Phase II IMvigor 210 study. ${ }^{39}$ To be eligible for cohort 2, patients were required to have progressive disease during or following therapy with a platinum-based chemotherapy regimen, or within 12 months of receiving neoadjuvant or adjuvant treatment. Atezolizumab was administered as a $1200 \mathrm{mg}$ IV infusion given on day 1 of each 3-week cycle, continuing until unacceptable toxicity, disease progression, or symptomatic progression occurs. PD-L1 status in tumor specimens was prospectively analyzed using a validated assay (Ventana SP142 assay by Roche) at a central laboratory. Patients were classified into two groups based on PD-L1 expression, using 5\% as cutoff. The primary end point of the study was ORR based on Resonse Evaluation Criteria in Solid Tumors (RECIST) 1.1 as well as the investigator-determined immune-modified RECIST. At a median follow-up of 14.4 months, ORR was $14.8 \%$ in the 310 treated patients, with higher ORR seen in the patients with high PD-L1 expression by immunohistochemistry (IHC) staining (26\%), though responses were noted even in the absence of tumor-infiltrating cells PD-L1 expression. Of the patients with a response, 17 had a $\mathrm{CR}(5.5 \%)$, including $5 \mathrm{CRs}$ in patients with PD-L1 expression $<5 \%$ and $12 \mathrm{CRs}$ in those with PD-L1 $\geq 5 \%$. The median duration of response was not reached, with response durations ranging from $2.1+$ to $13.8+$ months. At a median follow-up of 12 months, 38 of the 45 responses $(84 \%)$ were continuing.

Common treatment-related toxicities included fatigue $(52 \%)$, nausea $(25 \%)$, decreased appetite $(26 \%)$, pyrexia (21\%), and constipation (21\%), among others. ${ }^{40}$ In addition, $6.5 \%$ of patients required administration of corticosteroids for treatment of immune-related effects, and 2.3\% required thyroid hormone replacement. Three patients $(0.9 \%)$ expe-

Table I Summary of responses for immune checkpoint inhibitors in patients with advanced urothelial carcinoma

\begin{tabular}{|c|c|c|c|c|c|}
\hline Agent/study & Number of patients $(n)$ & ORR, \% & CR, \% & $\begin{array}{l}\text { Median OS, } \\
\text { months }\end{array}$ & $\begin{array}{l}\text { PD-LI diagnostic partner/ } \\
\text { Mab clone/Definition of PD-LI } \\
\text { expression for subgroup analysis }\end{array}$ \\
\hline \multicolumn{6}{|l|}{ Previously treated patients } \\
\hline $\begin{array}{l}\text { Atezolizumab, IMvigor } 210 \\
\text { (cohort 2), NCT02 I08652 }\end{array}$ & 310 & 14.8 & 5.5 & 7.9 & $\begin{array}{l}\text { Ventana/Rabbit SPI } 42 \\
I C 0(I C<I \%) \\
I C I(I C \geq I \% \text { and }<5 \%) \\
I C 2 / 3(I C \geq 5 \%)\end{array}$ \\
\hline $\begin{array}{l}\text { Pembrolizumab, KEYNOTE-045, } \\
\text { NCT02256436 }\end{array}$ & $\begin{array}{l}542 \text { ( } 270 \text { randomized to } \\
\text { pembrolizumab arm) }\end{array}$ & 21.1 & 7 & 10.3 & $\begin{array}{l}\text { Dako/Mouse } 22 \mathrm{C} 3 \\
\mathrm{PD}-\mathrm{LI} \geq 10 \%\end{array}$ \\
\hline $\begin{array}{l}\text { Nivolumab, CheckMate 032, } \\
\text { NCTOI } 928394\end{array}$ & 78 & 24.4 & 6 & 9.7 & $\begin{array}{l}\text { Dako/Rabbit 28-8 } \\
\text { PD-LI } \geq 1 \%\end{array}$ \\
\hline $\begin{array}{l}\text { Nivolumab, CheckMate 275, } \\
\text { NCT02387996 }\end{array}$ & 270 & 19.6 & 2 & 8.7 & $\begin{array}{l}\text { Dako/Rabbit } 28-8 \\
\text { PD-LI } \geq 1 \% \text { and } \geq 5 \%\end{array}$ \\
\hline Durvalumab, NCT0I693562 & 191 & 17.8 & 3.7 & I8.2 (immature) & $\begin{array}{l}\text { Ventana/Rabbit SP263 } \\
\text { PD-LI } \geq 25 \% \text { TC or IC }\end{array}$ \\
\hline Avelumab, NCT0I772004 & 44 & 18.2 & 11.4 & 13.7 & $\begin{array}{l}\text { Dako/Rabbit 73- } 10 \\
\text { PD-LI } \geq 5 \%\end{array}$ \\
\hline \multicolumn{6}{|l|}{ First-line cisplatin ineligible } \\
\hline $\begin{array}{l}\text { Atezolizumab, IMvigor } 210 \\
\text { (cohort I), NCT0295 I767 }\end{array}$ & 119 & 23 & 9 & 15.9 & $\begin{array}{l}\text { Ventana/Rabbit SPI } 42 \\
\text { ICO }(I C<I \%) \\
\text { ICI }(I C \geq I \% \text { and }<5 \%) \\
I C 2 / 3(I C \geq 5 \%)\end{array}$ \\
\hline $\begin{array}{l}\text { Pembrolizumab, KEYNOTE-052, } \\
\text { NCT02335424 }\end{array}$ & 370 & 29 & 7 & Not reported & $\begin{array}{l}\text { Dako/Mouse } 22 \mathrm{C} 3 \\
\mathrm{PD}-\mathrm{LI} \geq 10 \%\end{array}$ \\
\hline
\end{tabular}

Abbreviations: CR, complete response; OS, overall survival; PD-LI, programmed death-ligand I; ORR, overall response rate; IC, immune cell; TC, tumor cell; Mab, mono clonal antibody. 
rienced sepsis, intestinal obstruction, or pneumonitis that resulted in patient death.

Cohort 1 of the IMvigor 210 study also evaluated atezolizumab in the front-line setting for metastatic patients who were ineligible to receive cisplatin chemotherapy. ${ }^{41}$ One hundred nineteen patients were enrolled in this cohort of study, with renal dysfunction (83 patients) and suboptimal performance status ( 24 patients with ECOG 2 or worse) as the most common reasons for cisplatin ineligibility. At the 17.2 months median follow-up time, ORR was $23 \%$, with $9 \%$ CRs. The median response duration was not reached, with 19 of the 27 responders having continued treatment effect at the time of analysis. Responses did not correlate with the expression of PD-L1 in tumor-infiltrating lymphocytes. The median OS for cohort 1 was 15.9 months. Immune-related adverse events (AEs) were observed in $12 \%$ of patients, with 9 patients ( $8 \%$ ) discontinuing therapy due to treatmentrelated side effects. One treatment-related death occurred due to sepsis. Based on these data, atezolizumab was approved by the FDA in April 2017 for use in the up-front setting for patients who are not candidates for cisplatin chemotherapy.

The larger Phase III IMvigor 211 study was intended to validate the results of the prior Phase II study. In IMvigor $211,{ }^{42}$ the primary end point was an improvement in OS using atezolizumab for second-line therapy in locally advanced or metastatic bladder cancer. OS was to be tested in different groups based on PD-L1 expression, with statistical significance needing to be achieved in the immune cell (IC) $2 / 3$ group (highest level of PD-L1 expression) before evaluating OS in the study population as a whole. In the study, 931 previously treated patients were randomized to receive either atezolizumab or investigator's choice of chemotherapy, which included paclitaxel, docetaxel, or vinflunine. Though the final published results of the trial are still pending, a press release from Roche, the manufacturer of atezolizumab, in May 2017 showed that IMvigor 211 failed to meet its OS primary end point. ${ }^{43}$ While the treatment results for those receiving atezolizumab were similar to the Phase II study, the results seen in the chemotherapy arm were better than the planned study assumptions. The full published data from the study will be available in the future.

\section{Pembrolizumab (Keytruda, Merck, Inc)}

Pembrolizumab is a humanized PD-1 antibody, resulting in the blockade of both PD-L1 and PD-L2 ligands. The use of pembrolizumab in previously treated patients with metastatic urothelial carcinoma was evaluated in the KEYNOTE-045 study. ${ }^{44}$ This was a randomized, Phase III trial comparing pembrolizumab given at a dose of $200 \mathrm{mg}$ every 3 weeks to investigator's choice of either paclitaxel, docetaxel, or vinflunine, with PFS and OS as the co-primary end points of the study. PD-L1 status was assessed using the commercially available IHC 22C3 Dako assay with a cutoff of $10 \%$ or more. Five hundred forty two patients were enrolled in the study, with 270 receiving immunotherapy and the remaining 272 chemotherapy. In the pembrolizumab arm, OS was significantly increased at 10.3 months compared with 7.4 months with chemotherapy. In the subgroup with PD-L1 expression $\geq 10 \%$, median OS was 8.0 months compared with 5.2 months with other systemic therapy drugs. PFS was 2.1 months with pembrolizumab compared with 3.3 months with chemotherapy, which was not statistically significant. Overall response rates were higher in the immunotherapy arm as well (21\% vs $11 \%)$, including 19 patients (7\%) with a CR. Duration of response lasting for at least 12 months was also better with pembrolizumab (68\% compared with $35 \%$ with chemo). On subgroup analysis, there appeared to be greater benefit among current or former smokers compared with never smokers. AEs were noted in $69.1 \%$ of patients in the immunotherapy arm who were better tolerated with chemotherapy with at least $90 \%$ of patients reporting AEs. AEs were similar to other malignancies treated with pembrolizumab. Due to AEs, $5.6 \%$ of study patients discontinued treatment. The first high-level data confirming the benefit of immune checkpoint inhibitors over conventional chemotherapy comes from KEYNOTE-045, demonstrating a longer OS (by nearly 3 months) and better tolerability than chemotherapy.

Preliminary results of the single-arm, Phase II KEYNOTE-052 study were presented at the 2017 American Society of Clinical Oncology (ASCO) Meeting. The study included patients with advanced or metastatic urothelial carcinoma who were ineligible to receive cisplatin treatment. ${ }^{45}$ Renal impairment and poor performance status were the most common reasons for patients not being candidates for platinum-based therapy. Three hundred seventy patients with advanced disease received treatment with pembrolizumab, given at $200 \mathrm{mg}$ every 3 weeks for up to 2 years. The study population was older, with a median age of 74 years and a third of patients were over the age of 80 . The primary end point of the study was ORR. At a median follow-up of 9.5 months, ORR for the entire cohort was $29 \%$, including $7 \%$ CRs. At the time of analysis, the median duration of response had not yet been reached. Response rates were observed regardless of PD-L1 expression, though they did occur at a higher frequency in the PD-L1 high ( $\geq 10 \%)$ subgroup. The most common adverse reactions include fatigue, 
musculoskeletal pain, pruritis, nausea, loss of appetite, and diarrhea, among others. Eleven percent of patients discontinued therapy due to adverse reactions. Based on the efficacy demonstrated in the KEYNOTE-052 trial, pembrolizumab was granted accelerated approval for use in the first-line setting in cisplatin-ineligible patients in May 2017.

\section{Nivolumab (Opdivo, Bristol-Myers Squibb Co)}

Nivolumab is a fully human IgG4 antibody targeting PD-1. The efficacy of nivolumab was demonstrated in two studies. In CheckMate 032, patients with platinum-pretreated disease involving the renal pelvis, ureter, bladder, or urethra were given nivolumab at a dose of $3 \mathrm{mg} / \mathrm{kg}$ intravenously every 2 weeks. ${ }^{46}$ The primary end point of the study was ORR, assessed by investigator-evaluated RECIST 1.1. PD-L1 status was evaluated using the Dako IHC 28-8 pharmDx assay, using a cutoff of $1 \%$ for subgroup analysis, though it was done retrospectively and no cutoff was required to receive therapy on study. At the 15.2 months median follow-up time, ORR was $24.4 \%$, including five CRs (6\%). Treatment responses were observed regardless of PD-L1 expression. Though immune-related effects were not specifically reported, the most common adverse reactions included skin AEs (42\%) and gastrointestinal AEs (10\%). Two treatment-related deaths were noted, one due to thrombocytopenia and the other from pneumonitis. ${ }^{46}$

The larger single-arm, Phase II CheckMate 275 study included 270 patients with locally advanced, unresectable or metastatic disease that had progressed despite prior platinumbased therapy. ${ }^{47}$ ORR was again the primary end point. At the 7.0 months median follow-up time, ORR was $19.6 \%$, with results occurring independent of PD-L1 expression. PD-L1 level was measured using the Dako IHC 28-8 pharmDx assay, with $1 \%$ and $5 \%$ cutoffs. Median OS was 8.7 months; for those with PD-L1 above and below 1\%, survival rates were 6.0 and 11.3 months, respectively. Grade 3 or 4 AEs were recorded in $17.8 \%$ of patients, the most common being fatigue and diarrhea. ${ }^{47}$

\section{Durvalumab (Imfinzi,AstraZeneca)}

Durvalumab is an engineered, humanized PD-L1 antibody. Antibody-dependent cellular cytotoxicity is decreased via the creation of mutations in the Fc domain of the drug. ${ }^{48}$ Use of the treatment is indicated in patients with progressive disease during or following treatment with a platinum-based chemotherapy regimen, or within 12 months of receiving neoadjuvant or adjuvant treatment. Efficacy was demonstrated in the
Phase I/II 1108 study enrolling 191 pretreated patients (with all but one patient having received prior chemotherapy). ${ }^{49}$ PD-L1 expression was determined by the Ventana SP263 assay, with a cutoff of $25 \%$ staining in tumor or immune cells to determine high expression. Treatment was given at a dose of $10 \mathrm{mg} / \mathrm{kg}$ intravenously every 2 weeks. ORR with therapy was $17.8 \%$, including seven CRs (3.7\%). Response rate did correlate with PD-L1 expression, with an ORR of $27.6 \%$ for tumors with high expression compared with $5.1 \%$ for tumors with low or no expression. Median PFS and OS were 1.5 and 18.2 months (though OS data were considered immature at data cutoff), respectively, with 55\% survival rates at 1 year. Grade 3 or 4 treatment immune-related AEs were observed in $2.1 \%$ of patients. Two deaths occurred due to immune-related toxicities (pneumonitis, autoimmune hepatitis). Durvalumab received accelerated approval from the FDA in May 2017 for the treatment of metastatic urothelial carcinoma progressing during or after a platinum-based regimen. Durvalumab is currently being evaluated in the front-line setting regardless of platinum eligibility as a single agent and in combination with tremelimumab in the Phase III DANUBE trial (NCT02516241), comparing the study combination to standard of care platinum-based chemotherapy.

\section{Avelumab (Bavencio, EMD Serono/Pfizer)}

Avelumab is a fully humanized PD-L1 antibody. Conditional approval for the treatment was granted by the FDA in May 2017 for use in advanced urothelial cancer after progression on or after platinum-based therapy. This approval was granted based on a Phase Ib expansion cohort, in which 44 patients were treated with avelumab at a dose of $10 \mathrm{mg} / \mathrm{kg}$ IV given every 2 weeks, with the primary end point of safety and tolerability, with secondary end points of ORR by RECIST 1.1, PFS, OS, and PD-L1 associations with activity. PD-L1 status were determined by IHC using a Dako 73-10 assay, with tumors categorized based on intensity of PD-L1 staining in tumor membranes (with a threshold of 5\% in tumor cells). Treatment was given with a $10 \mathrm{mg} / \mathrm{kg}$ intravenous infusion of avelumab every 2 weeks. The first cohort of 44 patients was followed for a median of 16.5 months, demonstrating an $18.2 \%$ ORR, with best responses being five patients with a CR (11.4\%), three patients with a PR, and 15 patients with stable disease. While responses occurred in patients with prespecified PD-L1 positive and negative tumors (using a $5 \%$ cutoff), rates were notably higher in the PD-L1 positive tumors (53.8\% vs $4.2 \%$ in PD-L1 negative tumors). In patients who had a response, treatment effect was durable with continued effect in 6 of $8(75 \%)$ patients at the time of 
analysis. Median OS in the cohort was 13.7 months, with $54 \%$ of patients being alive at 1 year. The most commonly observed treatment-related effects included fatigue (31.8\%), infusion reactions (20\%), and nausea (11.4\%). Also, 20.5\% had immune-related toxicities, the most common being hypothyroidism, which occurred in $6.8 \%$ of patients. ${ }^{50}$ There were no treatment-related deaths.

\section{The changing landscape: incorporating immune checkpoint therapies into treatment for urothelial carcinoma}

There has been resurgence in treatment options in metastatic bladder cancer, after having been on a plateau for many years. With a multitude of novel therapies being approved, one of the main challenges is which agent to choose, and how to sequence them into our current treatment paradigm. Current therapeutic standards still prioritize cisplatin-based chemotherapy option as first-line therapy, with consideration of a non-cisplatin chemotherapy regimen or either atezolizumab or pembrolizumab for patients who have a contraindication to cisplatin. Following cisplatin-based chemotherapy, several checkpoint inhibitors have been approved, all with similar response rates and toxicity profile, with responses in the $20 \%$ range. Whether earlier utilization of immune checkpoint inhibitors would yield a survival advantage is an ongoing area of research, with clinical trials currently evaluating immune-oncology drugs not only in the first-line metastatic setting, but also in the adjuvant, neoadjuvant, and non-muscle-invasive disease as well. KEYNOTE-045 was the first Phase III randomized study to show greater benefit with the use of immune therapy drugs compared with taxane-based chemotherapy. ${ }^{42}$ At present, there are several ongoing Phase II and III studies evaluating immune therapy agents, either alone or in combination, as a first-line therapy compared to the current standard of care with a platinum-based chemotherapy regimen (Table 2).

Current standards still favor using platinum-based chemotherapy for treatment naïve patients, with typical response rates in the $40 \%-50 \%$ range. Responses may drop considerably going from the first- to second-line setting as cross-resistance between chemotherapy regimens occurs. As utilization of immune checkpoint inhibitors is moved to firstline therapy, whether cross-resistance occurs with administration of chemotherapy as next-line therapy after initial immune checkpoint inhibitors becomes particularly relevant. A recent study by Szabados et al provides some insight into whether cross-resistance occurs with changing sequencing practices. ${ }^{51}$ In this study, two different cohorts of sequential therapy were evaluated for patients with metastatic urothelial carcinoma, with 28 patients included in total. Cohort A consisted of 14 chemotherapy naïve patients, receiving front-line immune checkpoint inhibitors followed by chemotherapy at the time of disease progression, effectively evaluating the effects of chemotherapy after immunotherapy in metastatic disease. Cohort B was a chemotherapy-resistant group, receiving additional third-line chemotherapy after front-line standard chemotherapy followed by immunotherapy in the second line. The primary objective was response rates, as assessed by RECIST v1.1 criteria, in each cohort. Baseline patient characteristics were similar between the two groups. In cohort A, partial remission was achieved in nine patients (64\%), with three patients having stable disease and two having progressive disease leading to early death. PR was achieved in 3 patients $(21 \%)$ in cohort B, with 10 patients $(71 \%)$ having stable disease and 1 with disease progression. Overall, these results suggest a lack of cross-resistance between treatment types as evidenced by the maintenance of high response rates to chemotherapy after prior immune therapy drugs. ${ }^{51}$

Table 2 First-line immunotherapy trials in bladder cancer

\begin{tabular}{|c|c|c|c|c|}
\hline Study & NCT trial number & Treatment arms & Phase of study & $\begin{array}{l}\text { Estimated } \\
\text { completion date }\end{array}$ \\
\hline Danube & NCT025I624I & $\begin{array}{l}\text { Durvalumab + tremelimumab } \\
\text { versus durvalumab versus standard } \\
\text { chemotherapy }\end{array}$ & Phase II & July 15, 2019 \\
\hline IMvigor 130 & NCT02807636 & $\begin{array}{l}\text { Atezolizumab versus atezolizumab } \\
\text { in combination with platinum-based } \\
\text { chemotherapy versus platinum-based } \\
\text { chemotherapy }\end{array}$ & Phase III & July 30, 2020 \\
\hline KEYNOTE-36I & NCT02853305 & $\begin{array}{l}\text { Pembrolizumab with or without } \\
\text { platinum-based chemotherapy versus } \\
\text { platinum-based chemotherapy }\end{array}$ & Phase III & March 28, 2020 \\
\hline HCRN GUI0-I 48 & NCT0I52499I & $\begin{array}{l}\text { Ipilimumab in combination with } \\
\text { gemcitabine and cisplatin }\end{array}$ & Phase II & March 2018 \\
\hline
\end{tabular}


Another area of investigation is identification of a biomarker that could be predictive of response to immune therapy drugs. Typical ORRs for checkpoint inhibitors from published studies are in the range of $20 \%-25 \%$ at best. All of the current published trials have analyzed PD-L1 expression as a possible correlate, with some correlation in most studies, though it is not universally predictive and as many samples are from pretreatment biopsies and may not reflect dynamic changes that may develop throughout a patient's complex treatment course.

Rather than studying a single biomarker such as PD-L1, additional insights into disease behavior and clinical response may be provided through deeper molecular characterization of tumors. Prior analysis of muscle-invasive tumors confirms a diverse mutation spectrum with a high somatic mutation rate (median of 5.5 per megabase), ${ }^{52}$ on par with other diseases including melanoma and non-small cell lung cancer. Mutation signatures in the cytidine deaminase enzyme APOBEC are pervasive throughout bladder cancer genomes, reaching up to $68 \%$ of mutations in some samples, and contribute to this high mutational burden through early mutagenic activity. ${ }^{53}$ Using mRNA expression profiling and clustering, distinct molecular subtypes of bladder carcinoma can be identified, each of which may have different response rates to certain therapeutics. Using integrated RNA subtype classification, Robertson et al have proposed a stratification of five different subtypes of bladder cancer-luminal, luminal-papillary, luminal-infiltrated, basal/ squamous, and neuronal, with luminal and neuronal being newly described. ${ }^{54}$ The neuronal subtype, which accounted for approximately $5 \%$ of cases, had no histopathologic distinction from other specimens in most cases to aid in its identification, though expressed high frequencies of TP53 and RB1 mutations as seen in other types of small cell carcinomas. The luminal subtype, which was one of the most frequent subtypes noted constituting $35 \%$ of specimens tested, may have a low probability of response to neoadjuvant chemotherapy based on preliminary data from Seiler et al. ${ }^{55}$ Fibroblast growth factor receptor 3 mutations are common in the luminal subtype and may make a better therapeutic target for this population. The luminal-infiltrated type may also have a lower probability of response to neoadjuvant chemotherapy, though expresses CD270 (PD-L1) and CTLA4 immune markers and may yield better responses from an immune checkpoint inhibitor in this setting. ${ }^{54}$ Through use of mRNA expression-based subtype models, different molecular subtypes can be identified so that treatment decisions may be tailored to an individual based on molecular expression of disease, though will need to be validated in future clinical trials.

\section{Conclusion}

In summary, the recent incorporation of checkpoint inhibitors in the management of advanced bladder cancer has resulted in an expansion of treatment options for a difficult-to-treat disease. An ongoing focus of research within the field aims at how to best incorporate these agents in combination with other well-established therapeutics or earlier in the planned treatment course for patients. Continued clinical trials will better define the optimal roles these agents will play and may hopefully reveal better biomarkers or molecular profiles that may disclose which patients are most likely to benefit from such treatment options.

\section{Disclosure}

The authors report no conflicts of interest in this work.

\section{References}

1. Siegel RL, Miller KD, Jemal A. Cancer statistics, 2017. CA Cancer J Clin. 2017;67(1):7-30.

2. National Comprehensive Cancer Network. Clinical Practice Guidelines in Oncology: Bladder cancer, version 5. 2017. Available from: NCCN. org. Accessed December 13, 2017.

3. Park JC, Citrin DE, Agarwal PK, Apolo AB. Multimodal management of muscle invasive bladder cancer. Curr Probl Cancer. 2014;38(3):80-108.

4. Survival rates for bladder cancer. American Cancer Society. Available from: http://www.cancer.org/cancer/bladdercancer/detailedguide/ bladder-cancer-survival-rates. Accessed May 23, 2016.

5. Kirkwood JM, Butterfield LH, Tarhini AA, Zarour H, Kalinski P, Ferrone S. Immunotherapy of cancer in 2012. CA Cancer J Clin. 2012;62(5):309-355.

6. Intlekofer AM, Thompson $\mathrm{CB}$. At the bench: preclinical rationale for CTLA-4 and PD-1 blockade as cancer immunotherapy. J Leukoc Biol. 2013;94(1):25-39.

7. Logothetis CJ, Dexeus FH, Finn L, et al. A prospective randomized trial comparing MVAC and CISCA chemotherapy for patients with metastatic urothelial tumors. J Clin Oncol. 1990;8(6):1050-1055.

8. Tannock I, Gospodarowicz M, Connolly J, Jewett M.M-VAC (methotrexate, vinblastine, doxorubicin, and cisplatin) chemotherapy for transitional cell carcinoma: the Princess Margaret Hospital experience. J Urol. 1989;142(2 Pt 1):289-292.

9. Moore MJ, Iscoe N, Tannock IF. A phase II study of methotrexate, vinblastine, doxorubicin, and cisplatin plus recombinant human granulocyte-macrophage colony stimulating factors in patients with advanced transitional cell carcinoma. J Urol. 1993;150(4):1131-1134.

10. Sternberg CN, de Mulder P, Schornagel JH, et al; EORTC GenitoUrinary Cancer Group. Seven year update of an EORTC phase III trial of high-dose intensity M-VAC chemotherapy and G-CSF versus classic M-VAC in advanced urothelial tract tumours. Eur J Cancer. 2006;42(1):50-54.

11. von der Maase H, Hansen SW, Roberts JT, et al. Gemcitabine and cisplatin versus methotrexate, vinblastine, doxorubicin, and cisplatin in advanced or metastatic bladder cancer: results of a large, randomized, multinational, multicenter, phase III study. J Clin Oncol. 2000;18(17):3068-3077.

12. Galsky MD, Chen GJ, Oh WK, et al. Comparative effectiveness of cisplatin-based and carboplatin-based chemotherapy for treatment of advanced urothelial carcinoma. Ann Oncol. 2012;23(2):406-410.

13. Galsky MD, Hahn NM, Rosenberg J, et al. Treatment of patients with metastatic urothelial cancer "unfit" for cisplatin-based chemotherapy. J Clin Oncol. 2011;29(17):2432-2438. 
14. De Santis M, Bellmunt J, Mead G, et al. Randomized phase II/III trial assessing gemcitabine/carboplatin and methotrexate/carboplatin/ vinblastine in patients with advanced urothelial cancer who are unfit for cisplatin-based chemotherapy: EORTC study 30986. J Clin Oncol. 2012;30(2):191-199.

15. Sternberg CN, Calabro F, Pizzocaro G, Marini L, Schnetzer S, Sella A. Chemotherapy with an every 2 -week regimen of gemcitabine and paclitaxel in patients with transitional cell carcinoma who have received prior cisplatin-based therapy. Cancer. 2001;92(12):2993-2998.

16. Meluch AA, Greco FA, Burris HA 3rd, et al. Paclitaxel and gemcitabine chemotherapy for advanced transitional-cell carcinoma of the urothelial tract: a phase II trial of the Minnie pearl cancer research network. J Clin Oncol. 2001;19(12):3018-3024.

17. Li J, Juliar B, Yiannoutsos C, et al. Weekly paclitaxel and gemcitabine in advanced transitional-cell carcinoma of the urothelium: a phase II Hoosier Oncology Group study. J Clin Oncol. 2005;23(6):1185-1191.

18. Calabro F, Lorusso V, Rosati G, et al. Gemcitabine and paclitaxel every 2 weeks in patients with previously untreated urothelial carcinoma. Cancer. 2009;115(12):2652-2659.

19. Gitilitz BJ, Baker C, Chapman Y, et al. A phase II study of gemcitabine and docetaxel therapy in patients with advanced urothelial carcinoma. Cancer. 2003;98(9):1863.

20. Ardavanis A, Tryfonopoulos D, Alexopoulos A, Kandylis C, Lainakis G, Rigatos G. Gemcitabine and docetaxel as first-line treatment for advanced urothelial carcinoma: a phase II study. $\mathrm{Br} J$ Cancer. 2005;92(4):645-650.

21. Witte RS, Elson P, Bono B, et al. Eastern Cooperative Oncology Group phase II trial of ifosfamide in the treatment of previously treated advanced urothelial carcinoma. J Clin Oncol. 1997;15(2): 589-593.

22. Dreicer R, Gustin DM, See WA, Williams RD. Paclitaxel in advanced urothelial carcinoma: its role in patients with renal insufficiency and as salvage therapy. $J$ Urol. 1996;156(5):1606-1608.

23. Roth BJ, Dreicer R, Einhorn, LH, et al. Significant activity of paclitaxel in advanced transitional-cell carcinoma of the urothelium: a phase II trial of the Eastern Cooperative Oncology Group. J Clin Oncol. 1994;12(11):2264-2270.

24. de Wit R, Kruit WH, Stoter G, de Boer M, Kerger J, Verweij J. Docetaxel (Taxotere): an active agent in metastatic urothelial cancer; results of a phase II study in non-chemotherapy-pretreated patients. Br J Cancer. 1998;78(10):1342-1345.

25. McCaffrey JA, Hilton S, Mazumdar M, et al. Phase II trial of docetaxel in patients with advanced or metastatic transitional-cell carcinoma. $J$ Clin Oncol. 1997;15(5):1853-1857.

26. Lorusso V, Pollera CF, Antimi M, et al. A phase II study of gemcitabine in patients with transitional cell carcinoma of the urinary tract previously treated with platinum. Italian Co-operative Group on Bladder Cancer. Eur J Cancer. 1998;34(8):1208-1212.

27. Moore MJ, Tannock IF, Ernst DS, Huan S, Murray N. Gemcitabine: a promising new agent in the treatment of advanced urothelial cancer. $J$ Clin Oncol. 1997;15(12):3441-3445.

28. Bellmunt J, von der Maase H, Mead GM, et al. Randomized phase III study comparing paclitaxel/cisplatin/gemcitabine and gemcitabine/ cisplatin in patients with locally advanced or metastatic urothelial cancer without prior systemic therapy: EORTC Intergroup Study 30987. J Clin Oncol. 2012;30(10):1107-1113.

29. Gallagher DJ, Milowsky MI, Bajorin DF. Advanced bladder cancer: status of first-line chemotherapy and the search for active agents in the second-line setting. Cancer. 2008;113(6):1284-1293.

30. Sweeney CJ, Roth BJ, Kabbinavar FF, et al. Phase II study of pemetrexed for second-line treatment of transitional cell cancer of the urothelium. J Clin Oncol. 2006;24(21):3451-3457.

31. Ko YJ, Canil CM, Mukherjee SD, et al. Nanoparticle albumin-bound paclitaxel for second-line treatment of metastatic urothelial carcinoma: a single group, multicentre, phase 2 study. Lancet Oncol. 2013;14(8):769-76.
32. Bellmunt J, Theodore C, Demkov T, et al. Phase III trial of vinflunine plus best supportive care compared with best supportive care alone after a platinum-containing regimen in patients with advanced transitional cell carcinoma of the urothelial tract. J Clin Oncol. 2009;27(27):4454-4461.

33. Petrylak DP, de Wit R, Chi KN, et al; RANGE study investigators. Ramucirumab plus docetaxel versus placebo plus docetaxel in patients with locally advanced or metastatic urothelial carcinoma after platinumbased therapy (RANGE): a randomized, double-blind, phase 3 trial. Lancet. 2017;390(10109):2266-2277.

34. Glaser AP, Fantini D, Shilatifard A, Schaeffer EM, Meeks JJ. The evolving genomic landscape of urothelial carcinoma. Nat Rev Urol. 2017;14(4):215-229.

35. Rajasagi M, Shukla SA, Fritsch EF, et al. Systematic identification of personal tumor-specific neoantigens in chronic lymphocytic leukemia. Blood. 2014;124(3):453-462.

36. Lawrence MS, Stojanov P, Polak P, et al. Mutational heterogeneity in cancer and the search for new cancer-associated genes. Nature. 2013;499(7457):214-218

37. Brown SD, Warren RL, Gibb EA, et al. Neo-antigens predicted by tumor genome meta-analysis correlate with increased patient survival. Genome Res. 2014;24(5):743-750.

38. Herbst RS, Soria JC, Kowanetz M, et al. Predictive correlates of response to the anti-PD-L1 antibody MPDL3280A in cancer patients. Nature. 2014;515(7528):563-567.

39. Rosenberg JE, Hoffman-Censits J, Powles T, et al. Atezolizumab in patients with locally advanced and metastatic urothelial carcinoma who have progressed following treatment with platinum-based chemotherapy: a single-arm, multicenter, phase 2 trial. Lancet. 2016;387(10031):1909-1920.

40. Ning YM, Suzman D, Maher VE, et al. FDA approval summary: atezolizumab for the treatment of patients with progressive advanced urothelial carcinoma after platinum-containing chemotherapy. Oncologist. 2017;22(6):743-749.

41. Balar AV, Galsky MD, Rosenberg JE, et al. Atezolizumab as first-line treatment in cisplatin-ineligible patients with locally advanced and metastatic urothelial carcinoma: a single-arm, multicenter, phase 2 trial. Lancet. 2017;389(10064):67-76.

42. A Study of Atezolizumab Compared with Chemotherapy in Participants with Locally Advanced or Metastatic Urothelial Bladder Cancer [IMvigor211]. Available from: https://clinicaltrials.gov/ct2/show/ results/NCT02302807. Accessed December 13, 2017.

43. Genentech Provides Update on Phase III Study of Tecentriq (Atezolizumab) In People with Previously Treated Advanced Bladder Cancer. Available from: https://www.gene.com/media/pressreleases/14665/2017-05-09/genentech-provides-update-on-phase-iii-s. Accessed May 9, 2017.

44. Bellmunt J, de Wit R, Vaughn DJ, et al; KEYNOTE-045 Investigators. Pembrolizumab as second-line therapy for advanced urothelial carcinoma. NEnglJMed. 2017;376(11):1015-1026.

45. Balar AV, Castellano DE, O’Donnell PH, et al. Pembrolizumab as first-line therapy in cisplatin-ineligible advanced urothelial cancer: results from the total KEYNOTE-052 study population. J Clin Oncol. 2017;35(2).

46. Sharma P, Callahan MK, Bono P, et al. Nivolumab monotherapy in recurrent metastatic urothelial carcinoma (CheckMate 032):a multicenter, open-label, two-stage, multi-arm, phase 1/2 trial. Lancet Oncol. 2016;17(11):1590-1598.

47. Sharma P, Retz M, Siefker-Radtke A, et al. Nivolumab in metastatic urothelial carcinoma after platinum therapy (CheckMate 275):a multicenter, single-arm, phase 2 trial. Lancet Oncol. 2017;18(3):312-322.

48. Ibrahim R, Stewart R, Shalabi A. PD-L1 blockade for cancer treatment:MEDI4736. Semin Oncol. 2015;42(3):474- 483.

49. Powles T, O’Donnell PH, Massard C, et al. Efficacy and Safety of durvalumab in locally advanced or metastatic urothelial carcinoma: updated results from a Phase 1/2 Open-label Study. JAMA Oncol. 2017;3(9): e172411. 
50. Apolo AB, Infante JR, Balmanoukian A, et al. Avelumab, an anti-programmed death-ligand 1 antibody, in patients with refractory metastatic urothelial carcinoma: results from a multicenter, Phase Ib Study. J Clin Oncol. 2017;35(19):2117-2124.

51. SzabadosB, van Dijk N, Tang YZ, et al. Response rate to chemotherapy after immune checkpoint inhibition in metastatic urothelial cancer. Eur Urol. Epub 2017 Sep 13.

52. Lawrence MS, Stonjanov P, Mermel CH, et al. Discovery and saturation analysis of cancer genes across 21 tumour types. Nature. 2014;505(7484): 495-501.
53. Roberts SA, Lawrence MS, Klimczak LJ, et al. An APOBEC cytidine deaminase mutagenesis pattern is widespread in human cancers. Nat Genet. 2013;45(9):970-976.

54. Robertson AG, Kim J, Al-Ahmadie H, et al. Comprehensive molecular characterization of muscle-invasive bladder cancer. Cell. 2017;171(3): 540-556.

55. Seiler R, Ashab HAD, Erho N, et al. Impact of molecular subtypes in muscle-invasive bladder cancer of predicting survival after neoadjuvant chemotherapy. Eur Urol. 2017;72(4):544-554.

\section{Publish your work in this journal}

Research and Reports in Urology is an international, peer-reviewed, open access journal publishing original research, reports, editorials, reviews and commentaries on all aspects of adult and pediatric urology in the clinic and laboratory including the following topics: Pathology, pathophysiology of urological disease; Investigation and treatment of urological disease; Pharmacology of drugs used for the treatment of urological disease. The manuscript management system is completely online and includes a very quick and fair peer-review system, which is all easy to use. Visit http://www.dovepress.com/testimonials.php to read real quotes from published authors.

Submit your manuscript here: https://www.dovepress.com/research-and-reports-in-urology-journal 